\title{
Reformas, Relatos de Vida e Identidades Profesionales en Educación Especial: una Aproximación a Partir de las Voces de Educadores Especiales en Medellín (Colombia), 1965-2002
} REFORM, LifE Stories and PROFESSIONAL IDENTITY IN SPECIAL EDUCATION: AN APPROACH FROM THE VOICES OF SPECIAL EDUCATORS IN MEDELLIN (COLOMBIA)

\author{
Alexander YARZA DE LOS RÍOS ${ }^{1}$ \\ Myriam Ramírez RAMÍREZ ${ }^{2}$ \\ Lina María FRANCO 3 \\ Nancy Catalina VÁSQUEZ ${ }^{4}$
}

\begin{abstract}
RESUMEN: El artículo presenta los resultados de una investigación biográfico narrativo sobre las conexiones, relacionamientos y entrecruzamientos entre relatos de vida de maestros y maestras especiales y los procesos de reforma educativa a la educación especial en una ciudad de Colombia. Parte del reconocimiento de las historias de la educación especial en Colombia y América Latina, pero señalando la ausencia de investigaciones desde las voces y vivencias de los educadores especiales en el tiempo reciente, lo cual se corresponde con lo encontrado en el estado del arte. Posteriormente, se presentan parte de los resultados sobre el análisis de la identidad profesional docente especial y unas reflexiones para pensar el presente del educador especial/diferencial en América Latina.
\end{abstract}

PALABRAS CLAVES: Educación Especial. Investigación Biográfico-Narrativa. Identidad profesional. Historia de la Educación Especial.

\begin{abstract}
The article presents the results of a narrative biographical study on the connections, relationships and crosslinks between life stories of special teachers and educational special education reform process carried out in a city of Colombia. The paper first acknowledges the history of special education in Colombia and Latin America, while pointing out the lack of research on the voices and experiences of special educators in recent times, which corresponds with what is found in current studies. Next, partial results are presented: the analysis of special teacher professional identities and some reflections to enable us to think about special/differential educators in Latin America.
\end{abstract}

KEYWORDS: Special Education. Biographical Narrative Research. Professional Identity. History of Special Education.

\footnotetext{
${ }^{1}$ Lic. En Educación Especial. Magíster en educación, línea formación de maestros. Investigador del Grupo Historia de la Práctica Pedagógica en Colombia. Director del Grupo de Estudios e Investigaciones Sobre Educación Especial - GRESEE. victor.yarza@ udea.edu.co

${ }^{2}$ Socióloga. Magíster en educación. Investigadora del Grupo de Estudios e Investigaciones Sobre Educación Especial - GRESEE. myram19@gmail.com

${ }^{3}$ Lic. En Educación Especial. Mg. en educación y desarrollo humano. Investigadora del Grupo de Estudios e Investigaciones Sobre Educación Especial - GRESEE. linitafrancom@gmail.com

${ }^{4}$ Lic. En Educación Especial. Mg. en educación y desarrollo humano. Investigadora del Grupo de Estudios e Investigaciones Sobre Educación Especial - GRESEE. ncata91@hotmail.com
} 


\section{INTRODUCCIÓN}

La educación de las personas con discapacidad y excepcionalidad en América Latina tiene una multiplicidad de historias no contadas, invisibilizadas y sometidas, que esperan por ver la luz y resurgir mediante múltiples lenguajes. Existen contados esfuerzos académicos y políticos que se empeñan en trabajar arduamente, desde la historia de la educación especial, por recuperar y reescribir esa compleja trama de relaciones, sentidos, prácticas, instituciones, subjetividades, saberes, poderes, culturas, con todo lo que pueden enseñarnos en el presente y el futuro.

En la historiografía de la educación y la pedagogía latinoamericana, se aprecian dos intereses que requieren nuestra atención: por un lado, se viene girando fuertemente hacia el reconocimiento de las voces de los sujetos como una fuente válida, potente y significativa para la operación historiográfica. No sólo documentos legales, manuscritos, revistas, libros, informes de prensa, son suficientes para constituir los corpus documentales. El concepto de documento se amplía hacia otros umbrales, permitiendo recuperar las voces e incluso las imágenes (SANCHINDRIÁN BLANCO, 2011). De otro lado, aún se evidencia el poco interés por la educación especial como área u objeto de estudio histórico-pedagógico, aunque desde hace algún tiempo se viene trabajando asiduamente por cambiar este panorama. ${ }^{5}$

A lo largo de los diversos estudios e investigaciones, se ha entendido la importancia de vincular el análisis de las reformas educativas a los procesos de apropiación, circulación e institucionalización del saber pedagógico sobre los anormales, especiales, con discapacidad y excepcionalidad. Existen algunos estudios sobre las reformas a la educación para las personas con discapacidades y excepcionalidades en las últimas décadas en nuestra región latinoamericana, pero sin las voces de los sujetos, sin acercarse al análisis de la complejidad de estas reformas educativas, desde la perspectiva de uno de sus principales actores: los educadores especiales. $\mathrm{Al}$ respecto, como un ejemplo, se cuenta con el estudio Personas con discapacidad y acceso a los servicios educativos en Latinoamérica (SAMANIEGO, 2009), constituyéndose en una aproximación legal y conceptual, desde la perspectiva de informes internacionales y el discurso oficial de los distintos países latinoamericanos, en procura de respuesta a las demandas de inclusión de las personas con discapacidad al sistema educativo.

Las historias de la educación especial, educación o pedagogía diferencial son todavía escasas en Colombia (YARZA DE LOS RÍOS, 2007; 2011b) y en algunos países de América Latina (vg. Bolivia, Ecuador o Panamá). Como plantea en otro lugar Alexander Yarza De los

\footnotetext{
${ }^{5}$ En el año 2008, en el marco del Congreso "Ciencias, tecnologías y culturas. Diálogo entre las disciplinas del conocimiento. Mirando al futuro de América Latina y el Caribe”, realizado en la Universidad Santiago de Chile, se constituyó la mesa No 30 : Temas, problemas y nuevos desafios de la historia social de la educación, coordinada por Alicia Civera Cerecedo (Colegio Mexiquense, México), Lucía Lionetti (UNCPBA-FCH-IEHS, Argentina), Ariclé Vecchia (Universidad de Sao Paulo, Brasil), Carlos Escalante Fernández (El Colegio Mexiquense, México) y Alexander Yarza de los Ríos (Universidad de Antioquia, Colombia). Posterior a este encuentro, se gestaron dos iniciativas de publicación conjunta sobre las historias de la educación especial en América Latina. En 2010, el profesor Alexander Yarza De los Ríos coordinó un monográfico sobre Historias de la educación de anormales y la educación especial en América Latina, en la Revista Educación y Pedagogía (de la Universidad de Antioquia), en el cual se publicaron artículos provenientes de Argentina, Chile, Colombia, Brasil, España, México y Francia (una traducción). Posteriormente, en 2012 el profesor Antonio Padilla Arroyo, de la Universidad del Estado de Morelos, México, coordinó la edición del libro Arquetipos, memorias y narrativas en el espejo. Infancia anormal y educación especial en los siglos XIX y XX en donde se presentan varias aproximaciones históricas en Argentina, Colombia, Espańa y México. Para 2014 en el XI Congreso Iberoamericano de Historia de la Educación Latinoamericana, realizado en Toluca, México (6 y 9 de mayo), los profesores Padilla y Yarza coordinaron el Panel: Educación especial y prácticas educativas en Iberoamérica: imágenes y narrativas sobre el "cuerpo anormal", siglos XIX y XX, continuando con el trabajo de historización de la educación especial en América Latina.
} 
Ríos (YARZA DE LOS RÍOS, 2011b, p. 4): "En nuestra región (geopolíticamente hablando) todavía impera un olvido colectivo o una desmemoria conjunta de nuestra historia sobre la educación especial". Por tanto, todavía desconocemos buena parte de los procesos, dinámicas, estructuraciones, subjetivaciones, institucionalizaciones, apropiaciones, emergencias de discursos y prácticas relacionadas con la educación especial, la educación de sujetos/colectivos con discapacidad(es) y excepcionalidad(es) y, por supuesto, sobre el educador especial.

Con las historias de datos, institucionales, sociales y, especialmente, de la práctica pedagógica (YARZA DE LOS RÍOS, 2005; 2007; 2008; 2010, 2011a; 2011b; 2012; 2013; YARZA DE LOS RÍOS; RODRÍGUEZ, 2005, 2007; YARZA DE LOS RÍOS; CORTESE, 2009), se ha realizado un avance importante para el conocimiento de las historias de la educación especial en Colombia, desde principios del siglo XX a los albores del siglo XXI. Contamos con estudios sobre la institucionalización y apropiación de la pedagogía y educación de la infancia anormal, de los retrasados, de los "sordomudos", de los ciegos, en el contexto de los saberes modernos y el escolanovismo experimentalista, la problemática de la degeneración de la raza, la preocupación por el progreso y la salvación, entre otras temáticas y problemáticas que sitúan este discurso en el lugar epistémico de las ciencias modernas experimentales. De igual modo, tenemos aproximaciones histórico-comparadas a la educación de anormales entre Colombia y Argentina (1900-1930), estudios sobre la formación de maestros en y para la educación de anormales (1870-1940), acercamientos institucionales a Escuelas de ciegos y sordos (1925-2000), miradas histórico sociales sobre la educación especial, con referencia a Bogotá (1900-1995) y un conjunto menor de miradas generalistas, normativas, prescriptivas y globales (1900-1990).

Los enfoques epistémicos y metodológicos de estos estudios han prescindido de considerar las voces de los sujetos históricos, de sus narraciones y relatos en/de vida, como un documento y un registro histórico (en el sentido moderno de la historiografía), al tiempo que tampoco se ha contemplado su potencia constructiva, reconstructiva, mediadora y hasta transgresora de las verdades en la educación y la pedagogía, específicamente en el campo de la educación especial.

Las voces singulares, cuando se entrelazan, tienen la potencia de tejer un relato compartido sobre nuestra historia común. En un país con olvido y amnésico como Colombia, todos los intentos por reconstruir los pasos y trasegares de nuestra historia educativa y pedagógica, por tanto social y cultural, deben entenderse como un esfuerzo por reinventar el presente para proyectarnos hacia otro futuro. Pensar históricamente, entonces, como dice la historiadora mexicana María Esther Aguirre Lora:

[...] propiciaría otra forma de teorizar, de inteligir, que superaría la inmediatez y el presentismo [...] que aún domina el campo de los estudios en educación, al ofrecer una perspectiva crítica que apunte a la historicidad de los distintos problemas que forman parte del campo de estudios educativos, esto es, como se han constituido cada uno de ellos en el tejido de las distintas temporalidades, cuáles son las diversas trayectorias que se entrecruzan en ellos (AGUIRRE LORA, 2009, p. 29).

En esta dirección, la investigación biográfico narrativa estudió las conexiones, relacionamientos y entrecruzamientos entre relatos de vida de educadores y educadoras especiales y los procesos de reforma a la educación especial, constituyéndose en una apuesta profesional, ético-política y académica del equipo de investigación y de las narradoras y el 
narrador, en tanto ha generado posibilidades de comprensión de lo que ha sido la historia de la educación especial en los tiempos recientes, desde el punto de vista singular y colectivo de los educadores especiales (no de las leyes ni desde un externalismo sin sujetos).

\section{INVESTIGANDO BIOGRÁFICA Y NARRATIVAMENTE EN LA EDUCACIÓN ESPECIAL}

La investigación en el campo de la educación para personas con discapacidad y excepcional o campo de la educación especial (YARZA DE LOS RÍOS, 2011b), ha tenido expresiones de distintos enfoques y paradigmas investigativos: etnográficos, investigación acción participación, cuasi-experimentales y psicopedagógicos (FRANCO et al., 2011). No obstante, prescinde de acercamientos sistemáticos, rigurosos y profundos, desde un enfoque (auto) biográfico-narrativo a la cotidianidad y vida educativa en nuestras sociedades contemporáneas. Por tanto, la investigación parte de la identificación de la ausencia de investigaciones biográfico narrativas, específicamente sobre los educadores especiales, la identidad y las reformas educativas en Colombia y, en un modo incipiente, en América Latina.

De otro lado, desde el conjunto de los múltiples y diversos modelos de análisis de las reformas educativas (APPLE, 2002; POPKEWITZ, 1994; POPKEWITZ; TABACHNICK; WEHLAGE, 2007), se evidencia la urgencia y necesidad de comprender los procesos de reforma y transformación política desde las experiencias y vivencias de los actores en concreto, desde los sujetos de las reformas. Una situación equivalente sucede en el conjunto de investigaciones en historias y relatos de vida del profesorado, en tanto avanzan sobre las narrativas de educadores de educación inicial, básica (primaria y secundaria), media, universidad, pero existen ínfimos acercamientos focalizados en los educadores especiales.

Siguiendo la presentación al Monográfico sobre Narrativas, autobiografías y formación, de la Revista Educación y Pedagogía (de la Universidad de Antioquia), que hizo el investigador argentino Daniel Hugo Suárez (2011), podemos apreciar la reciente expansión del giro (auto) biográfico narrativo en educación y pedagogía: congresos, foros, seminarios, libros, dossiers en revistas, trabajos de pregrado, maestría y tesis doctorales, redes, publicaciones gubernamentales, etc. Esta expansión permite afirmar la existencia de una "sociedad biográfica", de un "espacio biográfico" y del reconocimiento de la inminencia de los procesos de biografización en la cultura contemporánea.

Después de una amplia revisión de revistas, libros, congresos, trabajos de maestría y tesis doctorales en Colombia y en América Latina, se constató la escasa existencia de estudios biográfico-narrativos sobre la identidad profesional del educador especial y sus conexiones con las reformas educativas para las personas con discapacidad y excepcionalidad. De la mano de los análisis y perspectivas de McEwan y Egan (2005), Delory Momberger (2009), Passegi (2011), Bolívar Botía (2002), Bolívar Botía, Fernández Cruz y Segovia (2001), Bolívar Botía y Segovia (2006), Bolívar Botía, Gallego, León y Pérez (2005), Rivas et al. (2012) y De Tezanos (2012), se identifican una multiplicidad de temas, líneas y "objetos" de investigación, formación y acción en la investigación narrativa: profesorado, adultos, inmigrantes, aprendices, interculturalidad, conflicto, sexualidad, ruralidad, memoria, epistemología y metodología de la investigación, prácticas de formación, género, infancia, cuerpos, salud, cuidado de sí, lenguaje y narrativa, familia, artes, literatura, imágenes, documentación narrativa, vulnerabilidad, trayectorias 
intelectuales y profesionales, educación inicial, básica, media y superior, entre otras. En este contexto general, se investiga poco sobre discapacidad, educación y educadores especiales.

Como ejemplos de lo encontrado, se pueden citar, por su gran trayectoria, impacto y alcance internacional, los Congresos Internacionales de Pesquisa (Auto) Biográfica de Brasil: CIPA. A 2012 se han realizado cinco versiones de este importante evento que convoca una gran cantidad de investigadores, de distintas partes del mundo y en donde se visibilizan las temáticas, tendencias y orientaciones de la investigación, la formación y la acción. Con la revisión del V CIPA (2012), se identificó un trabajo sobre jóvenes mujeres con discapacidad (COBEÑAS, 2012) y un trabajo sobre narrativas de profesoras de educación especial (VARELA BLANCO, 2012). A partir de este último trabajo, se pudo conocer el estudio pionero de Henrique Silva (2005), Bittencourt Leitão (2008) y Henrique Silva, Pino Sirgado y Vasques Tavira (2012), los cuales se convierten en referentes fundamentales y orientadores de la presente investigación. De otro lado, en los Simposios Internacionales de Narrativas en educación, subjetividad y formación (2011 y 2013), solo se encontraron nuestros aportes (FRANCO, 2011; YARZA et al., 2012).

Para el caso de Chile, contamos con los trabajos de la pedagoga Silvia López de Maturana (2010), que han contribuido a comprender los rasgos de los "buenos maestros", en su compromiso pedagógico por un proyecto educativo, desde las historias de sus vidas profesionales. En un estudio posterior, con un equipo de investigación (LÓPEZ DE MATURANA, 2012), profundizaron sobre las prácticas pedagógicas de maestros y directivos chilenos, desde sus autobiografías y entrevistas narrativas en profundidad. Al otro lado del atlántico, José Ignacio Rivas y Fernando Hernández vienen liderando investigaciones narrativas en educación, a partir de lo cual problematizan las reformas educacionales, la constitución de subjetividades, el lugar de investigador, la identidad profesional, entre otras temáticas (RIVAS FLORES, 2011; RIVAS et al. 2010; HERNÁNDEZ; SANCHO; RIVAS, 2011; RIVAS et al., 2012).

En la Colección "Narrativas, Autobiografías y Educación”, publicada por el Consejo Latinoamericano de Ciencias Sociales, coordinada por Daniel Hugo Suárez, de la Universidad de Buenos Aires, ninguno de los libros o capítulos hace referencia a nuestro tema de estudio, aunque sus contribuciones teóricas son imprescindibles para la investigación (DELORY-MOMBERGER, 2009; PASSEGI; DE SOUZA, 2010; ALLIAUD; SUÁREZ, 2011). De parte de la colección "Pesquisa (auto)biográfica $\infty$ Educação" y de los libros de la Red Biograph, Associação Brasileira de Pesquisa (Auto)Biográfica, de Brasil, donde existe una amplia cantidad de publicaciones ${ }^{6}$, no se encuentra ninguna referencia a educadores especiales, identidad y reformas.

Para el caso de Colombia, desde hace poco más de una década se puede apreciar el giro (auto)biográfico narrativo en el trabajo con los maestros y maestras de las escuelas de nuestro país. Las experiencias recopiladas por la Expedición Pedagógica Nacional (Expedición pedagógica nacional, 2001), por el Instituto para la Investigación Educativa y el Desarrollo Pedagógico (1999), por la Fundación Social (1998) o por Rodrigo Parra Sandoval (PARRA SANDOVAL, 1986; PARRA SANDOVAL; PARRA; LOZANO, 2006), entre otros, se constituyen en unos antecedentes significativos para el trabajo de historias y relatos de vida de educadores especiales. De igual manera, desde la investigación educativa y pedagógica sobre

${ }^{6}$ Para ampliar sobre este asunto visitar la página: http://www.biograph.org.br/index.php?option=com_content\&view=category $\&$ layout $=$ blog $\&$ id $=38 \&$ Itemid $=58$ 
la formación de maestros y el oficio de enseñar se han realizado diferentes acercamientos a las historias de vida de maestros (DÍAZ MEZA, 2007; RUEDA MEZA, 2008).

En la Facultad de Educación de la Universidad de Antioquia, se vienen realizando interesantes acercamientos a las prácticas y experiencias de maestros de Medellín y Antioquia desde la narrativa, los relatos y las historias de vida (DUARTE; SOTO; MURILLO, 2007; SUÁREZ; YARZA, 2008; MURILLO ARANGO, 2008, 2014), así como la coordinación de los Simposios Internacionales de Narrativas en educación, subjetividad y formación (referenciados anteriormente). No obstante, ninguna de estas experiencias, investigaciones o proyectos se ha concentrado en indagar sobre las experiencias de educadores especiales y sus múltiples relaciones con las reformas educativas y la identidad profesional.

En nuestra búsqueda documental, los estudios auto-biográfico narrativos sobre la profesión docente y los estudios sobre la profesionalidad y profesión docente, en Colombia, Iberoamérica, Estados Unidos y Europa (BIDDLE; GOOD; GOODSON, 2000; ESTEVE ZARAZAGA, 2006; VELAZ DE MEDRANO; VAILLANT, 2009; IBARRA MUNOZZ, 2010; NUÑEZ ROJAS; ARÉVALO VERA;ÁVALOS DAVIDSON, 2012; IMBERNÓN MUÑOZ, 2012), se concentran en los educadores de primaria, secundaria e incluso de los profesores universitarios e investigadores, prescindiendo de estudios a la singularidad del educador especial en Colombia y en América Latina (como ya se señaló y con la salvedad de Brasil). Como si en la luz del día de la profesión docente no existiese otra noche y otra luz: una narrativa especial que hunde sus raíces sobre la "normalidad docente".

Finalmente, en el importante estado del arte de los trabajos de maestría y tesis doctorales en educación y pedagogía en Colombia, entre 2000 y 2010, coordinado por los Doctores Napoleón Murcia Peńa y Héctor Fabio Ospina (2012), la temática no se referencia ni como tema ni como prospectiva de trabajo; de hecho, se señala la precariedad de estudios en educación especial y discapacidad en Colombia ${ }^{7}$.

En síntesis, en el campo disciplinar de la investigación auto biográfica narrativa (como la nombra PASSEGI, 2011), se encuentra un mínimo (aunque importante) antecedente de investigación y se constata un escaso interés por estudiar la identidad profesional del educador especial y sus conexiones con las reformas a la educación de las personas con discapacidad y excepcionalidad, así como un bajísimo uso y problematización de la narrativa en el campo de la discapacidad.

\footnotetext{
${ }_{7}$ Así aparece la "ínfima existencia investigativa” en las Maestrías y Doctorados en educación y pedagogía en Colombia: “Región investigativa: Modalidades educativas. $12 \%$ de la producción nacional. Subregión: Educación especial 2,3\%. Las subregiones Educación en primera infancia y preescolar, Educación especial, Etnoeducación, Educación para la crianza, Educación y arte, y Educación y medios audiovisuales reportan bajos porcentajes de participación en la producción investigativa en el nivel nacional. Se quiere resaltar, muy especialmente, el tema de la Educación en primera infancia y preescolar dadas las condiciones sociales y políticas con las que se cuenta para darle prioridad al tema en los trabajos de investigación en los niveles de formación posgraduada en educación y pedagogía en el país." (MURCIA PEÑA Y OSPINA, 2012, p. 149)
} 


\section{TRAYECTORIAS DE LA “IDENTIDAD PROFESIONAL DOCENTE ESPECIAL”: UN ACERCAMIENTO POLÍTICO PEDAGÓGICO Y BIOGRÁFICO NARRATIVO}

A partir de los análisis colectivos y cruzados de las historias de vida de las narradoras y el narrador ${ }^{8}$, fue emergiendo la posibilidad de temporalizar y periodizar temáticamente las narraciones en una trama polifónica que vincula cada historia singular (por tanto: irreductible, irrepetible, insustituible) con una historia colectiva, común, pública, compartida. El paso de lo individual a lo colectivo, desde las historias de vida, nos permite mostrar la trama y el entramado histórico, desde el que se erige la historicidad de la subjetividad de los educadores especiales, de los procesos educativos y pedagógicos especiales (con su inminente hibridismo entre lo pedagógico, médico, psicológico y social), de las tensiones con el Estado y con las agencias internacionales, del lugar social de la educación especial, de las representaciones sociales de los niños/niñas especiales y con discapacidad/excepcionalidad, de las relaciones con la familia, la comunidad y el profesorado (de preescolar, primaria y secundaria), de las relaciones interdisciplinarias, interinstitucionales e intersectoriales, de la vida cotidiana en las aulas, las escuelas, los barrios y la ciudad, de las vidas íntimas y públicas de unos maestros y maestras especiales, diferentes, radicalmente distintos de otros profesores y maestros de nuestra educación, en nuestra Colombia y, por extensión, en América Latina.

En medio de esa trama polifónica y en contraste con el análisis sobre la desprofesionalización del educador especial planteado por el investigador mexicano Guajardo Ramos (2012), nuestro estudio sobre la identidad profesional docente especial se vio fortalecido por las investigaciones del español Antonio Bolívar y su equipo (BOLÍVAR; DOMINGO, 2005, 2009), específicamente su análisis sobre la identidad profesional de profesores de secundaria y las reformas escolares; con el argentino Gustavo Mórtola y su estudio sobre la identidad laboral docente de profesores de primaria (MÓRTOLA, 2010); con el trabajo de Araceli de Tezanos (2012), sobre identidad y tradición docente; y, finalmente, el pionero trabajo en Brasil de Daniel Nunes Henrique Silva (2005) sobre memorias y narrativas en educación especial en Rio de Janeiro y sus reflexiones posteriores (HENRIQUE SILVA; PINO SIRGADO; VASQUES TAVIRA; 2012; VARELA BLANCO, 2012), además de la investigación de Maria Elisa Bittencourt Leitão (2008) sobre trayectorias de vida y de formación académica de educadores especiales, en la ciudad de Vitória, en el Estado de Espíritu Santo. En este fructífero encuentro académico, se pudo robustecer la conceptualización sobre la identidad profesional con el pionero y vigente análisis sobre la identidad del educador especial del investigador francés Paul Fustier (1972/2009). En el fondo, se encuentran las reflexiones transdisciplinares y poscoloniales del intelectual Stuart Hall (2003).

Sintéticamente, la identidad profesional docente no es una esencia inherente al individuo o un elemento externo e impuesto a la conducta del sujeto, sino que es un proceso continuo de reconstrucción y reelaboración de la mirada hacia el yo, desde el sí mismo y desde el otro. En tanto proceso es dinámico, interactivo, relacional. La construcción de identidad implica auto y co-referenciación del sujeto en función de si, de los otros, de los campos profesionales-disciplinares y de la sociedad y cultura (en un momento histórico).

\footnotetext{
${ }^{8}$ En esta investigación el proceso de selección y convocatoria dio como resultado la oportunidad de contar en total con 17 participantes narradores, de los cuales fueron 16 educadoras y un educador especial, distinguidos en tres grupos: entre 8 y 15 ańos, entre 15 y 30 años, más de 30 años, teniendo en cuenta la importancia de experiencia desde el punto de vista generacional.
} 
En la perspectiva de Hall (2003), la identidad se construye, no se pre-establece ni prefabrica, al tiempo que se deconstruye y reconstruye, en un juego de intermediaciones sociales, culturales, de lenguaje, históricas, personales e institucionales, las cuales, en nuestro caso, también se encuentra vinculada a una profesión, un campo de saber, con sus prácticas, unas relaciones pedagógicas, unos sujetos y unas instituciones concretas, todas girando alrededor de la "educación especial". La identidad profesional se ve afectada por los procesos sociales de reforma educativa (BOLÍVAR; DOMINGO, 2005, 2009), conllevando incluso a dinámicas de re-profesionalización o des-profesionalización.

La profesionalización, desprofesionalización y reprofesionalización, junto con los procesos de constitución de la identidad profesional docente especial (IPDE), se encuentran estrechamente vinculados y condicionados a los procesos de estatalización y desestatalización/ privatización de la educación especial, en tanto espacialidad/institucionalidad (es decir, instituciones, escuelas y aulas) y servicio/oferta escolarizada desde el Estado.

Evocando las metáforas del lúcido sociólogo Zigmun Bauman (2003), en la “época dorada" de estatalización de la educación especial, se constituye una IPDE “sólida”, múltiple y estable. Con la creación de las aulas y escuelas especiales entre 1960 y 1980, y su fortalecimiento en el contexto de la Escuela Expansiva en América Latina y Colombia (MARTÍNEZ BOOM, 2004; 2009), las educadoras especiales con una larga trayectoria contaron con la posibilidad de una vinculación contractual-laboral permanente con el Estado (municipio o Departamento), lo cual permitió que fueran cobijadas por un Estatuto Docente progresista en la región latinoamericana (Estatuto 2277 de 1978). El estatuto docente y un conjunto de resoluciones que otorgaban estímulos económicos adicionales (primas, sobresueldos, incentivos, entre otros), establecieron unas condiciones materiales para la naciente profesión de educador especial en el país, en comparación con maestros de otros grados o niveles. Las condiciones económicas afectaban positivamente la IPDE.

De otro lado, la IPDE también se vio condicionada por la baja cantidad de alumnos especiales (a pesar de sus "enormes dificultades" intelectuales, sensoriales, comportamentales, etc.), el apoyo constante de equipos interdisciplinarios de profesionales para-pedagógicos, los procesos de cualificación, capacitación y profesionalización permanentes, la construcción de saber pedagógico y didáctico especializado, la programación curricular articulada, sólida y fundamentada (en las teorías y en las prácticas psicomotrices-conductuales y constructivistas), lineamientos académicos claros, válidos y universales (guías, documentos ministeriales, cartillas, etc.), aunada a una contundente elección vocacional (desde el inicio de la profesión o como un efecto secundario producto del azar) y una voluntad pasional por la educación del otro con discapacidad, en tanto expresión de la alteridad deficiente.

Estas primeras educadoras especiales continuarán fortaleciendo su IPDE en el seno del enfoque psicomotriz conductual, en las distintas instituciones y a través de una cantidad innumerable de experiencias educativas, formativas y escolares, articuladas a los sujetos y los contextos socio-culturales. De igual modo, aconteció con el tránsito hacia el constructivismo y/o cognitivismo y los enfoques interculturales o psicopedagógicos, de la década de 1990 y 2000.

Con el giro estratégico de la década de 1990, esa ola de reformas legislativas a la educación, en una tensión entre las demandas internacionales y el movimiento pedagógico 
nacional colombiano, estas educadoras especiales comenzaron a resignificar su identidad profesional a partir del advenimiento de los discursos integracionistas plasmados en la nueva Ley General de Educación, de 1994, y su decreto reglamentario 2082 de 1996 (pero, efectivamente en la cotidianidad y en la formación venían fraguándose desde mediados de 1980).

Durante esta década, algunas educadoras empezaron a verse (autoidentificación) como maestras de apoyo a los procesos de integración escolar desde sus instituciones educativas especializadas y en una nueva conexión sistémica con otras escuelas, llamadas regulares u ordinarias. El cambio en la función profesional y la "fusión/desaparición" de las escuelas especiales como servicio/oferta/modalidad estatalizada, reprofesionalizó a las educadoras especiales en los espacios de educación escolarizada en nuestra sociedad, sin necesariamente erosionar la "solidez identitaria”. En este grupo de maestras, se aprecia una reprofesionalización que resignifica y reconstruye la identidad profesional, posibilitando la incorporación en sus discursos y prácticas de nuevos enfoques pedagógicos, didácticos, psicológicos, sociales, etc., la utilización y acercamiento a otros discursos y perspectivas epistemológicas (bilingüismo, interculturalidad, enfoque antropológico, para el caso de los Sordos, por ejemplo) e, incluso, en alguna maestra, la construcción de orientaciones para la trasformación de las escuelas y aulas comunes, en función de la integración.

En la investigación, también se pudo constatar la emergencia o surgimiento de una IPDE “fluida”, ligada al conjunto de los gestos de desestatalización/privatización”, re y desprofesionalización del educador especial en el contexto de las reformas neoliberales de la década del "giro estratégico". Las educadoras y el educador con mediana y corta trayectoria, desde su formación inicial universitaria, se encontraron de frente con los procesos de reprofesionalización en contextos de integración escolar, entre el "desmonte" y la "fusión", y ante una desprofesionalización que genera enrarecimientos y modificación en la IPDE.

Algunas vivieron intensamente la transformación de las aulas y escuelas especiales en nuevas instituciones o su desaparición lenta, pero certera: se transformaban en escuelas integradoras. Todo esto sin contar con una vinculación contractual-laboral estable con el Estado y percibiendo la existencia de una distancia entre sus fines iniciales de formación (por vocación o azar) y el ejercicio profesional. Las funciones del educador de apoyo a la integración empiezan a co-existir con las propias del "antiguo" educador especial y con el cierre y apertura de nuevos espacios institucionales, como las Aulas de Apoyo Especializado o las Unidades de Atención Integral (que prestan sus servicios y asesoramientos a los directivos, docentes y familias para garantizar la inclusión en las escuelas comunes).

En este nuevo escenario reformista (vertical y global), los educadores especiales se verán en el centro de una crisis (como señala Fustier). Y, desde allí, en la cotidianidad, reconstruyen y resignifican su identidad profesional docente especial, a pesar de la tercerización del servicio, la precarización del trabajo y la profesión, e incluso, de la jubilación (de las maestras con más trayectoria). En todo caso, las narraciones nos han permitido constatar que los procesos de reforma educativa activados desde la década de 1990, han ido "desestatalizando" las diversas

\footnotetext{
${ }^{9}$ Los tres gestos de desestatalización son: Gesto 1. Desaparición de los internados (década de 1980); gesto 2: Desmonte de la División de Educación Especial y el equipo interdisciplinario (década de 1990); gesto 3: Fusión de escuelas especiales en instituciones educativas (en el marco de la integración escolar y la educación inclusiva, década de 2000).
} 
formas de institucionalización de la educación especial, hasta desmontarla y transformar las instituciones; lo cual no ha representado, en todos los casos, beneficios para los alumnos, las familias y los mismos maestros y maestras. Todo lo anterior también se vio reflejado y diferenciado en los modelos de formación de maestros que se fortalecieron a principios de la década de 1980 y existen hasta la actualidad, viviendo los propios cambios que iban en sincronía con las reformas educativas a las personas con discapacidad y excepcionalidad.

Podemos plantear, en términos generales, que un hallazgo importante del estudio consiste en evidenciar que la reforma educativa vertical y globalizada de la década de 1990 y principios de 2000, no produce una prescripción absolutista sobre la existencia singular de los educadores especiales, a pesar de las transformaciones de las condiciones externas a su identidad profesional. Existe una multiplicidad de formas de construirse como educador especial. Más allá de las prescripciones de los Estatutos docentes, de las funciones escolares, de las reglamentaciones ministeriales o de las "teorías exógenas", ha emergido una heterogeneidad de devenires, de contingencias de ser educador especial en nuestro contexto (que también nos permitiría entender algunos asuntos en América Latina).

Los educadores especiales recrean, rehacen o reinventan las directrices gubernamentales, produciendo una resignificación táctica en sus espacios institucionales. En algunos casos de agudización del verticalismo de la reforma de integración (década de 1990), los maestros se constituyeron en una subjetividad política de resistencia, bien fuera desde la movilización social, la alianza con las familias o desde la militancia en los sindicatos de Medellín y Antioquia. $\mathrm{Y}$, al mismo tiempo, existieron algunos maestros que se adaptaron, adecuaron y asumieron a-críticamente, los nuevos planteamientos. Como plantea Paul Fustier (2009), en la crisis y en los conflictos que se presentan en la cotidianidad escolar se configura la identidad del educador especial; así también, en la verticalización de la reforma se produjeron dos modulaciones de identidad profesional colectiva, como opciones a los educadores especiales, en su individualidad: a-crítica y crítica.

\section{Por UN PEDAgogo (POlítico) ESPECIAL: UN SUEÑo A MÚltiPleS VOCES}

Desde hace poco más de una década, la literatura utilizada para la formación de educadores especiales y, en términos generales, algunos tratados, investigaciones y posiciones globalizadas sobre integración y la educación inclusiva, procedentes de países europeos (principalmente España) y anglo-norteamericanos traducidos al habla hispana y portuguesa, plantean contundentemente que la educación especial había "fracasado" como servicio institucionalizado. ${ }^{10}$ Esta perspectiva se oficializa en la medida que se naturaliza como verdad en la formación inicial y continuada de maestros y en la investigación, en el contexto de la escolarización inclusiva.

Desde los organismos internacionales y el agenciamiento operado por la "función experto" (MARTÍNEZ BOOM; OROZCO TABARES, 2011), se esperaría que la educación especial en Colombia hubiese sido objeto de un diagnóstico que derivase en un conjunto de

\footnotetext{
${ }^{10}$ Hace pocos años, el investigador Alfredo Sarmiento (2010) presentó un análisis pormenorizado y extenso sobre la calidad de la educación inclusiva en Colombia, con especial énfasis en discapacidad. Literalmente concluye el informe que "Las personas con discapacidad tienen un retraso de cerca de dos décadas de avances educativos".
} 
medidas para remediar su crisis o estado deficitario. No obstante, lo que se evidencia es un continuum de reformas aceleradas, verticalistas, descontextualizadas y sin diálogo de saberes con los actores-educadores, familias y sujetos con discapacidad y excepcionalidad ${ }^{11}$. En esta dirección, las voces de los sujetos que fueron vistos como objetos o pieza secundaria en el proceso de reforma a la educación especial, se erigen para narrar otra historia, una contrahistoria que intenta desnaturalizar las miradas occidentales que asumimos en la formación de maestros y en el campo de la educación especial (en general). Una historia que se abre a la pluralidad de sentidos y construcciones de micro-políticas educativas que tienen sus lenguajes, prácticas y modos de subjetivación. Una contrahistoria que deja entrever una contra-diagnosis de nosotros mismos desde otro prisma.

Más acá de las prescripciones históricas oficializadas sobre la educación especial que marcan su ineludible fracaso, las historias de vida nos muestran su indiscutible vitalidad pedagógica, la presencia de una pregunta fundamental por los umbrales de formabilidad y por la enseñanza y educación integral/armónica que nos permiten afirmar que estas educadoras y educador son productoras de saber pedagógico y didáctico, de una cultura escolar inédita y transformadoras de nuestras realidades de exclusión, segregación y marginación.

En otra dirección, consideramos fundamental, necesario y pertinente avanzar hacia la comprensión de los procesos de diferenciación estructural profesional del educador especial o diferencial en América Latina: la multiplicidad de la profesión en el seno de las reformas neoliberales de la educación y en las contiendas asociadas al imperativo de la inclusión. Los trabajos existentes en Brasil sobre los educadores especiales, desde la investigación biográfico narrativo, nos permite entablar un diálogo de saber intercultural Sur-Sur, en dónde se puedan desentrañar y reconfigurar las tramas polifónicas de la vida cotidiana de estos educadores y su multiplicidad constitutiva, reivindicando la potencia que tiene la reinvención de la identidad profesional en tiempos líquidos, de riesgo, de modernidad tardía, transmodernidad, postcapitalismo o globalización.

La pregunta por la identidad profesional y la tradición, nos sitúa de cara al fortalecimiento/redimensión de la formación inicial y continua de educadores especiales o diferenciales en nuestras instituciones de educación superior. Y, de igual modo, nos ubica en un horizonte de trabajo conjunto, en redes y colaborativo, en torno a una Comunidad Político Académica del Sur que tiene como uno de sus propósitos la construcción sistemática de saber pedagógico y didáctico en relación con la educación de las personas con discapacidad y excepcionalidad, en un vínculo estrecho con el co-diseño de alternativas de escolarización, educación, formación y atención integral, y con la defensa radical de sus derechos y de nuestros derechos ${ }^{12}$.

\footnotetext{
${ }^{11}$ Hace pocos años, el investigador Alfredo Sarmiento (2010) presentó un análisis pormenorizado y extenso sobre la calidad de la educación inclusiva en Colombia, con especial énfasis en discapacidad. Literalmente concluye el informe que "Las personas con discapacidad tienen un retraso de cerca de dos décadas de avances educativos".

${ }^{12}$ En América Latina contamos con dos experiencias de larga data, con un trabajo colaborativo, sistemático y permanente. Para el caso de la Argentina, tenemos el caso de la Red Universitaria de Educación Especial y para el caso de Brasil la Asociación Brasilera de Investigadores Sobre Educación Especial. La Red y la Asociación cuentan con mecanismos constantes de diálogo y comunicación disciplinar, como Congresos, Encuentros o Revistas, al tiempo que una representación amplia de sus países.
} 
Tal vez, de esta manera, con estos sentidos, más otros que tejamos y conversemos juntos, se pueda entender que una "identidad profesional docente especial" se erige como una condición de posibilidad para un pedagogo (político) especial. En la sutura de auto-reconocerse y co-reconocerse como un sujeto de saber emerge una subjetividad política, transformadora, de contraconductas y resistencias que sin duda alguna continuará cambiando nuestra historia de exclusiones, expulsiones, discriminaciones, invisibilizaciones, desde la educación, la formación y la pedagogía.

\section{REFERENCIAS}

AGUIRRE LORA, M. E. Lo que la Historia nos puede decir de la Diferencia. En: MEDINA, P. Epistemologias de la diferencia. Debates contemporáneos sobre la identidad en las prácticas educativas. México: Plaza y Valdés, p. 23-50, 2009.

ALLIAUD, A.; SUÁREZ, D. El saber de la experiencia. Narrativa, investigación y formación docente. Buenos Aires: CLACSO, 2011.

APPLE, M. W. Mercados. Estándares y desigualdad: ¿pueden las pedagogías críticas parar las políticas derechistas?. Revista de Educación, Número Extraordinario, Madrid, 2002. p. 223-248.

BAUMAN, Z. Modernidad líquida. México: FCE, 2003.

BIDDLE, B. J.; GOOD, T. L. y GOODSON, I. F. La enseñanza y los profesores. España: Paidós, 2000.

BITTENCOURT LEITÃO, M. Professores que atuam na educação especial: trajetórias de vida e de formação acadêmica. 2008. Dissertação (Mestrado em Educação) - Programa de Pós-Graduação em Educação, Universidade Federal do Espírito Santo, Vitória, 2008.

BOLÍVAR BOTÍA, A. ¿De nobis ipsis silemus?: Epistemología de la investigación biográfico-narrativa en educación”. Revista electrónica de Investigación Educativa, v. 4, n. 1. Disponible en http://redie. uabc.mx/vol4no1/contenido-bolivar.html Acceso en 15 de feb. 2014.

BOLÍVAR BOTÍA, A.; FERNÁNDEZ CRUZ, M. y SEGOVIA, J. D. La investigación biográficonarrativa en educación. Madrid: La Muralla, 2001.

BOlÍVAR BOTÍA, A; GALlEGO, M. J.; LEÓN, M. J.; PÉREZ, P. Políticas Educativas de Reforma e Identidades Profesionales: El Caso de la Educación Secundaria en España”. Education Policy Analysis Archives/Archivos Analiticos de Politicas Educativas, Arizona, v. 13, p. 1-51. 2005. Disponible en: http:// www.redalyc.org/src/inicio/ArtPdfRed.jsp?iCve=275020513045 Acceso en 30 mar. 2014.

BOLÍVAR BOTÍA, A.; SEGOVIA, J. D. La investigación biográfico narrativa en Iberoamérica: Campos de desarrollo y estado actual. Forum: Qualitative Social Research, Berlín, v. 7, n. 4, 2006 Disponible en: http://www.qualitative-research.net/index.php/fqs. Acceso en 15 feb. 2014.

BOLÍVAR BOTÍA, A.; FERNÁNDEZ CRUZ, M.; MOLINA RUIZ, E. Investigar la identidad profesional del profesorado: Una triangulación secuencial”. Forum Qualitative Sozialforschung / Forum: Qualitative Social Research, Berlín, v. 6, n. 1, Art. 12, 2005. Disponible en: http://nbn-resolving.de/ urn:nbn:de:0114-fqs0501125. Acceso en 15 feb. 2014.

COBEÑAS, P. Reflexiones metodológicas sobre la investigación de autopercepciones de jóvenes mujeres con discapacidad. V CONGRESSO INTERNACIONAL DE PESQUISA (AUTO) BIOGRÁFICA - V CIPA. Porto Alegre, PUCRS: Casa Leiria, 2012. 
DE TEZANOS, A. ¿Identidad y/o tradición docente? Apuntes para una discusión. Revista Perspectiva Educacional, Viña del Mar, v. 51, n. 1, p. 1-28, 2012. Disponible en: http://www. perspectivaeducacional.cl/index.php/peducacional/article/viewFile/71/29 Acceso en 17 abr. 2014.

DELORY-MOMBERGER, C. Biografía y educación. Figuras del individuo-proyecto. Buenos Aires: CLACSO, 2009.

DÍAZ MESA, C. Narrativas docentes y experiencias escolares significativas: Relatando el sentido de ser maestro. Guillermo de Ockham, Cali, v.5, n.2, p.55-65, 2007.

ECHEITA, G.; DOMÍNGUEZ, A. Educación inclusiva. Argumento, caminos y encrucijadas. AULA revista de pedagogía de la Universidad de Salamanca, Salamanca, v.17, p.25-35, 2011.

ECHEITA, G; PARRILLA, A.; CARBONELL, F. La educación especial a debate. Revista RUEDES. Red Universitaria de Educación Especial, Mendoza, v.1, n.1, p.35-53, 2011. Disponible en: http:// bdigital.uncu.edu.ar/3594. Acceso en 15 may. 2014.

EXPEDICIÓN PEDAGÓGICA NACIONAL. Preparando el equipaje. Bogotá: Universidad Pedagógica Nacional, 2001.

ESTEVE ZARAZAGA, J. M. La profesión docente en Europa: perfil, tendencias y problemática: la formación inicial. Revista de Educación, Madrid, n.340, p.19-86, 2006.

FRANCO, L. et al. Educación especial como campo de saber e investigación biográfico-narrativa. SIMPOSIO INTERNACIONAL NARRATIVAS EN EDUCACIÓN, SUBJETIVIDAD Y FORMACIÓN. Medellín: Universidad de Antioquia, 2011.

FUNDACIÓN SOCIAL. Historias de maestros: La vida un quehacer matemático y el abuelo. Bogotá: Fundación Social, 1998.

FUSTIER, P. L'identité de l'éducateur spécialisé. Francia: Dunod, 2009.

GOODSON, I. Historias de vida del profesorado. España: Octaedro, 2001.

GUAJARDO RAMOS, E. La desprofesionalización docente en educación especial. Revista

Latinoamericana de Inclusión Educativa, Santiago de Chile, v. 4, n. 1, p. 105-126, 2012. Disponible en: http://www.rinace.net/rlei/numeros/vol4-num1/art5.pdf. Acceso en 7 abr. 2014.

HALL, S. ¿Quién necesita la identidad? En: HALL, S. y DU GAY, P. (eds). Cuestiones de Identidad. Buenos Aires: Amorrortu Editores, 2003.

HENRIQUE SILVA, D. N. Educação especial: memórias e narrativas docentes. Rio de Janeiro: Secretaria Municipal do Rio de Janeiro, 2005.

HENRIQUE SILVA, D. N.; PINO SIRGADO, A.; VASQUES TAVIRA, L. Memória, narrativa e identidade profissional: analisando memoriais docentes. Revista Cadernos Cedes, Campinas, v.32, n. 88, p.263-283, 2012.

HERNÁNDEZ, F.; SANCHO, J. M. y RIVAS, J. I. (coord.) Historias de vida en educación. Biografias en contexto. Barcelona: Esbrina-Recerca, 2011.

IBARRA RUSSI, O. A. Ser de maestro en Colombia: de oficio a profesión: perspectiva histórica, social y pedagógica de las transformaciones de la actividad educadora en nuestro país. Bogotá: Universidad Pedagógica Nacional, 2010.

IMBERNÓN MUÑOZ, F. La investigación sobre y con el profesorado. La repercusión en la formación del profesorado, ¿cómo se investiga? Revista Electrónica de Investigación educativa, Baja 
California, v.14, n.2, p.1-9, 2012. Disponible en: http://redie.uabc.mx/vol14no2/contenidoimbernon2012.html

INSTITUTO PARA LA INVESTIGACIÓN EDUCATIVA Y EL DESARROLLO PEDAGÓGICO. Violencia en la escuela, Vidas de maestros. Bogotá, IDEP, 1999.

LÓPEZ DE MATURANA, S. Los buenos profesores. Educadores comprometidos con un proyecto educativo. La serena: Editorial Universidad de La Serena, 2010.

LÓPEZ DE MATURANA et al. ¿Cómo son los profesores que educan a nuestros hijos? La Serena: Editorial Universidad de La Serena, 2012.

MARTÍNEZ BOOM, A. De la escuela expansiva a la escuela competitiva. Dos modos de modernización en América Latina. Barcelona, Bogotá: Anthropos, CAB, 2004.

MARTÍNEZ BOOM, A. La educación en América Latina: un horizonte complejo. Revista Iberoamericana de Educación, Madrid, n.49, p.163-179, 2009.

MARTÍNEZ BOOM, A.; OROZCO, H. Políticas de escolarización en tiempos de multitud. Revista Educación y Pedagogía, Medellín, v.22, n.58, p.105-122, 2010.

MCEWAN, H.; EGAN, K. (comp) La narrativa en la enseñanza, el aprendizaje y la investigación. Buenos Aires: Amorrortu, 2005.

MÓRTOLA, G. Enseñar es un trabajo. Construcción y cambio de la identidad laboral docente. Buenos Aires: Noveduc, 2010.

MURCIA, N.; OSPINA, H. Regiones investigativas en educación y pedagogía en Colombia: construcción de un mapa de la actividad investigativa de tesis de Maestría y Doctorados en el período 2000-2010. Manizales: CINDE-Universidad de Manizales, 2012. Disponible en: http://biblioteca.clacso.edu.ar/Colombia/alianza-cinde umz/20130316014724/

RegionesinvestigativasenEducacionyPedagogiadetesisdeMaestriasyDoctoradosenColombia.pdf. Acceso en 5 de jun. 2014.

MURILLO, G. (Comp.) Maestros contadores de historias: relatos de vida. Medellín: Gobernación de Antioquia, Secretaria de Educación para la Cultura. Artes y letras, 2008.

MURILlO, G. (Comp.) Relatos de vida. Buen Comienzo. Medellín: Universidad de Antioquia. Programa Buen Comienzo, 2014.

NÚNEEZ ROJAS, M. A.; ARÉVALO VERA, A.; ÁVALOS DAVIDSON, B. Profesionalización docente: ¿Es posible un camino de convergencia para expertos y novatos? REDIE. Revista Electrónica de Investigación Educativa, Baja California, v.14, n.2, p.10-24, 2012.

PADILLA ARROYO, A. (Coord.) Arquetipos, memorias y narrativas en el espejo. Infancia anormal y educación especial en los siglos XIX y XX. Morelos: Universidad Autónoma del Estado de Morelos, 2012. PARRA SANDOVAL, R. Los maestros colombianos. Bogotá: Plaza \& Janes, 1986.

PARRA SANDOVAL, R.; PARRA, F.; LOZANO, M. Tres talleres: hacia una pedagogía de la investigación etnográfica en la escuela. Bogotá: Convenio Andrés Bello, 2006.

PASSEGI, M. Aproximaciones teóricas a las perspectivas de la investigación (auto)biográfica en educación. Revista Educación y Pedagogía, Medellín, v.23, n.61, p.25-40, 2011.

PASSEGI, M.; DE SOUZA, E. Memoria docente, investigación y formación. Buenos Aires: CLACSO, 2010. 
POPKEWITZ, T. Sociología política de las reformas educativas: el poder/saber en la enseñanza, la formación del profesorado y la investigación. Madrid: Morata, 1994.

POPKEWITZ, T.; TABACHNICK, B.; WEHLAGE, G. El mito de la reforma educativa. Un estudio de las respuestas de la escuela ante un programa de cambio. Barcelona-México: Pomares Corredor, 2007.

RIVAS, J. Historias de vida y emancipación: subjetividad, conflicto y cambio social. Diálogos: Educación y formación de personas adultas, España, n. 3, p. 67-68, 2011.

RIVAS, J. et al. La configuración de identidades en la experiencia escolar. Escenarios, sujetos, regulaciones. Revista de Educación, Madrid, n. 353, p. 187-209, 2010.

RIVAS, J. et al. Historias de vida en educación: sujeto, diálogo, experiencia. Barcelona: ProCie, 2012.

RUEDA MEZA, J. Historia de maestros para maestros. Pedagogía narrativa expresada en relatos de vida. Bogotá: Universidad de la Salle, 2008.

SAMANIEGO, P. Personas con discapacidad y acceso a servicios educativos en Latinoamérica. Breve análisis de situación. España: CERMI, 2009.

SANCHIDRIÁN BLANCO, C. El uso de imágenes en la investigación histórico-educativa. Revista de Investigación Educativa, Murcia, v.29, n.2, p.295-309, 2011.

SARMIENTO, A. Situación de la Educación en Colombia. Preescolar, Básica, Media y Superior. Bogotá: Fundación Saldarriaga Concha, 2010.

SUÁREZ, J.; YARZA, A. Maestros de las Escuelas normales superiores de Antioquia "Historias de Vida". Medellín: Gobernación De Antioquia. Secretaría de Educación para la Cultura. Colección Memoria, 2008.

SUÁREZ, D. H. Narrativas, autobiografías y educación. Una presentación y algunos comentarios. Revista Educación y Pedagogía, Medellín, v.23, n.61, p.11-22, 2011.

VARELO BLANCO, L. de M. Contando de si e encantando os demais: narrativas de professoras de educacao especial. En: CONGRESSO INTERNACIONAL DE PESQUISA (AUTO) BIOGRÁFICA CIPA, 5.; Porto Alegre, PUCRS: Casa Leiria, 2012.

VELAZ DE MEDRANO, C.; VAILLANT, D. Aprendizaje y desarrollo profesional docente. España: OEI. Metas 2021, 2009.

YARZA DE LOS RÍOS, A. Travesías: notas para una pedagogía y una epistemología de la educación especial en Colombia. Revista de Pedagogía, Caracas, v.26, n.76, p.281-306, 2005.

YARZA DE LOS RÍOS, A. Algunos modos de historiar la educación especial en Colombia: una mirada crítica desde la historia de la práctica pedagógica. Revista Brasileira de Educação Especial, Marília, v. 13, n. 2, p. 173-188, 2007.

YARZA DE LOS RÍOS, A. Infancia escolarizada, menores anormales y retrasados: apuntes para una historia comparada de las reformas instruccionistas, el correccionalismo y la educación de anormales en Antioquia y Buenos Aires, 1870-1938. XIV CONGRESO COLOMBIANO DE HISTORIA, 14., Tunja. Universidad Pedagógica y Tecnológica de Tunja, 2008.

YARZA DE LOS RÍOS, A. Del destierro, el encierro y el aislamiento a la educación y la pedagogía de anormales en Bogotá y Antioquia. Principios del siglo XIX a mediados del siglo XX. Revista Educación y Pedagogía, Medellín, v.22, n.57, p.111-129, 2010. 
YARZA DE LOS RÍOS, A. Educadores especiales en la educación inclusiva como reforma y práctica de gubernamentalidad en Colombia: ¿perfil, personal o productor de saber?. Revista Brasilera Currículo Sem Fronteiras, Brasil, v.11, n.1, p.34-41, 2011a. Disponible en: http://www.curriculosemfronteiras. org/vol1 1 iss 1 articles/rios.htm Acceso en 20 jun. 2014.

YARZA DE LOS RÍOS, A. Corrientes pedagógicas, tradiciones pedagógicas y educación especial Pensando históricamente la educación especial en América Latina. Revista RUEDES: de la Red Universitaria de Educación Especial, Mendonza, v. 1, n. 1, p. 3-21, 2011b. Disponible en: http:// bdigital.uncu.edu.ar/fichas.php?idobjeto=3582. Acceso en 25 may. 2014.

YARZA DE LOS RÍOS, A. Cursos de perfeccionamiento y de información pedagógica sobre enseñanza de anormales y pruebas mentales y de instrucción en Buenos Aires (Argentina) y Antioquia (Colombia), 1926-1939. En: PADILLA ARROYO, A. (Coord.). Arquetipos, memorias y narrativas en el espejo. Infancia anormal y educación especial en los siglos XIX y XX. Morelos: Universidad Autónoma del Estado de Morelos, 2012.

YARZA DE LOS RÍOS, A. Hacer sitio al que llega: Pedagogía, Educación Especial y Formación. Revista Contextos de educación, Río Cuarto, v.13, n.14 2013. Disponible en: http://www.hum.unrc. edu.ar/publicaciones/contextos/articulos/vol14/pdfs/04-yarza.pdf Acceso en 13 jun. 2014.

YARZA DE LOS RÍOS, A.; CORTESE, M. Análisis de los procesos de apropiación y emergencia de la educación o pedagogía de anormales en Medellín y Buenos Aires, 1900-1920: un estudio histórico exploratorio de educación comparada. Informe final de investigación. Sede de Investigación Universitaria. Medellín. Universidad de Antioquia, 2009.

YARZA DE LOS RÍOS, A.; RODRÍGUEZ, L. (2005) Horizonte conceptual y tecnologías médicopsico-pedagógicas en la "pedagogía de anormales" en Colombia: 1920-1940. Revista Educación y Pedagogía, Medellín, v.17, n.41, p.55-68, 2005.

YARZA DE LOS RÍOS, A.; RODRÍGUEZ, L. Educación y pedagogía de la infancia anormal. 18701940. Contribuciones a una historia de su apropiación e institucionalización en Colombia. Bogotá: GHPP. Cooperativa Editorial Magisterio, 2007.

YARZA, A. et al. "Contrahistorias" de la educación especial en Medellín (Colombia): historias de vida y reformas educativas.” En: SIERRA AGUDELO, G. (Comp.) Necesidades educativas especiales. Intervención pedagógica, psicológica y familiar. Medellín: Editorial Corporación Ser Especial, 2012. p. 231-249.

Recebido em: 07/07/2014

Reformulado em: 17/09/2014

Aprovado em: 18/09/2014 